\title{
Antifungal Drug Resistance among Candida albicans and Non-albicans Candida Species Isolates from a Tertiary Care Centre at Allahabad
}

\section{Singh $M$ and Chakraborty $A^{*}$}

Department of Microbiology, Moti Lal Nehru Medical College, Allahabad, U.P, India

*Corresponding author: Arindam Chakraborty, Asst. Professor, Department of Microbiology Moti Lal Nehru Medical College, Allahabad, U.P, India, Tel: +917408881369; E-mail: arindam_cy@yahoo.com

Received date: September 18, 2017; Accepted date: October 16, 2017; Published date: October 18, 2017

Copyright: (c) 2017 Singh M, et al. This is an open-access article distributed under the terms of the Creative Commons Attribution License, which permits unrestricted use, distribution, and reproduction in any medium, provided the original author and source are credited.

\begin{abstract}
Objective: The aim of our study was to identify the distribution of Candida species among clinical isolates and their sensitivity pattern for common antifungal drugs.

Methods: This descriptive study was carried out in a tertiary care hospital in Allahabad. A total of One hundred and four Candida isolates were included in the study. Identification and speciation of the isolates were carried out by Germ-tube test, Chlamydoconidia production test, colony characterization in chromogenic agar medium, carbohydrate assimilation test and growth at $45^{\circ} \mathrm{C}$. Four antifungal drugs such as Fluconazole (25 mcg), Clotrimazole $(10 \mathrm{mcg})$, Nystatin $(100 \mathrm{U})$ and Amphotericin-B (100 U) were tested by disk diffusion method. Descriptive statistics was used which involves the use of simple percentage and bar chart to analyze the data. In addition, Chi-square test was performed and $\mathrm{P}$ value was calculated.

Results: Out of 104 Candida isolates, 60 (58\%) were from Urine, 36 (34.5\%) were from Sputum and 8(7.5\%) were from blood isolates. Of the total of 104 isolates 64 (61.5\%) were Candida albicans, 20(19\%) Candida dubliniensis, 10 (9\%) Candida tropicalis, 7(6.5\%) Candida parapsilosis and 4(4\%) Candida glabrata respectively. Resistance rates for commonly use antifungal drugs among the isolates are as followed Fluconazole 76 (73\%), Amphotericin B 40 (38.5\%), Nystatin 4(4\%) and Clotrimazole 60 (58\%) respectively. For Candida albicans and Candida dubliniensis most active antifungal drug was Nystatin (93\%; $100 \%$ respectively).
\end{abstract}

Discussion: Species level identification of Candida and their antifungal sensitivity testing should be performed routinely in all microbiology laboratories to prevent the spread of antifungal resistance.

Keywords: Antifungal susceptibility; Non-albicans Candida; Candida dubliniensis

\section{Introduction}

Candida is major human fungal pathogens that cause both mucosal and deep tissue infections. In recent years, the incidence of Candida infection has increased largely because of the rising rate of immune suppressive therapy [1] and also enhances the infection with multidrug resistant bacteria which leads to overuse of higher classes of antibiotic and as a result there is a destruction of commensal bacteria, owing to this there is an increased opportunity of Candida to cause infection in those patients. The treatment of such Candidal infections is a major concern since they are showing a high rate of anti-fungal drug resistance.

For many years Candida albicans is the predominant cause of invasive candidiasis in the majority of clinical settings, nevertheless, the epidemiology of Candida infection has changed in recent years, with longitudinal studies reporting that a considerable proportion of patients are now infected with non-albicans candida (NAC) species. Thus the potential clinical importance of species-level identification has been recognized as Candida species differ in the expression of putative virulence factors and antifungal susceptibility $[2,3]$.
In India, there is insufficient data regarding the Candida species and their susceptibility pattern [4] hence, the present study was undertaken to identify different Candida species and their sensitivity pattern with the available antifungal agents.

\section{Material and methods}

This prospective study was conducted during the period from November 2016 to April 2017 from patients of the tertiary care hospitals in Allahabad after obtaining permission from the institutional ethical committee. One hundred and four strains of Candida species with significant growth were isolated from the specimen such as clean catch midstream urine, blood and sputum using standard sterile procedures from the study population. Those subjects who had received antifungal drugs during the past one month, Urine and sputum samples shows insignificant growth of Candida species and polymicrobial infections were excluded.

\section{Isolation and identification}

Blood and sputum sample was processed in Blood agar and MacConkey Agar and Urine sample were processed on MacConkey Agar and CLED (Cystine lactose electrolyte deficient) Agar. Suspected colonies of Candida were farther cultured on Sabouraud dextrose agar with chloramphenicol (SDA, HiMedia). The isolates were identified 
Citation: Singh M, Chakraborty A (2017) Antifungal Drug Resistance among Candida albicans and Non-albicans Candida Species Isolates from a Tertiary Care Centre at Allahabad . J Antimicrob Agents 3: 150. doi:10.4172/2472-1212.1000150

Page 2 of 4

based on colony morphology, Gram staining and by standard biochemical properties which include:

\section{Germ-tube test}

Yeast cells were inoculated into $0.5 \mathrm{~mL}$ of pool serum and incubated at $37^{\circ} \mathrm{C}$ for 2.5 hour. After this period, aliquots were removed for microscopic examination. Germ tube was considered as a slender tube with straight walls, without septum and without constriction at the junction between the cells [5].

\section{Chlamydoconidia production test}

Chlamydoconidia production test was performed using corn meal agar medium. The samples previously grown in SDA were seeded as 3 parallel streaks in a rectangular piece of corn meal agar placed between two slides, incubated in the wet chamber at $30^{\circ} \mathrm{C}$ for $72 \mathrm{~h}$ and visualized in an optical microscope (10X and 40X magnification). The formation of rounded spores with double-wall isolates was observed as chlamydoconidia and was indicative of Candida albicans or Candida dubliniensis [6].

\section{Chromogenic agar culture}

Each isolate was cultured on SDA at $30^{\circ} \mathrm{C}$ for 48 hour. After this, they were seeded on HiCrome Candida Differential agar (Hi Media, Mumbai) and incubated at $30^{\circ} \mathrm{C}$ for $48 \mathrm{hr}$. The CHROMAgar ${ }^{\text {mat }}$ allows selective yeast isolation, identifying colonies of Candida albicans, Candida dubliniensis, Candida tropicalis, Candida glabrata and Candida krusei on the basis of colour and colony morphology. The strains were identified according to the manufacturer's instructions, which define light green colonies as Candida albicans, purple fuzzy colonies as Candida krusei, steel blue to blue as Candida tropicalis and cream to white as Candida glabrata [7].

\section{Carbohydrate assimilation test}

The carbohydrate assimilation test determines the ability of a yeast isolate to use a particular carbohydrate substrate as its sole carbon in a medium. Yeasts were cultured in YPD for $24 \mathrm{~h}$ at $30^{\circ} \mathrm{C}$. After the cultures were centrifuged for $5 \mathrm{~min}$ at $2500 \mathrm{~g}$ and the pellets were washed 3 times successively, suspended in sterile saline and adjusted to 5 McFarland standards. A volume of $300 \mu \mathrm{L}$ of Yeast Nitrogen Base agar (Hi Media, Mumbai, India) and $1.5 \mathrm{~mL}$ of Candida sp. suspension was mixed with $30 \mathrm{~mL}$ of sterile bacteriological agar in Falcon tubes. The suspension was placed in $15 \mathrm{~cm}$ Petri dishes and after solidification of the medium, discs with the $2 \%$ carbohydrates (maltose, trehalose, xylose, galactose, lactose, sucrose and glucose) were placed. The suspension was incubated at $30^{\circ} \mathrm{C}$ for $96 \mathrm{hr}$, and observed daily. Glucose was used as a positive control since all the species of Candida assimilate this carbohydrate. The carbohydrate assimilation was observed with a presence of a halo of growth around each carbohydrate [6,7].

\section{Growth at $45^{\circ} \mathrm{C}$}

Growth at $45^{\circ} \mathrm{C}$ has been considered a useful test for the differentiation of Candida dubliniensis (no growth) from Candida albicans (growth). This test was used in the positive samples for the germ-tube test, for chlamydoconidia production and that developed green colonies in HiCrome agar. The temperature test was performed in SDA (with Antibiotic) medium. All samples were incubated at $45^{\circ} \mathrm{C}$, and growth was assessed daily for 10 days. 6

\section{Antifungal sensitivity testing}

Antifungal susceptibility testing of the isolates was performed by four antifungal drugs disk Fluconazole $(25 \mathrm{mcg})$, Clotrimazole (10 mcg), Nystatin (100 U) and Amphotericin-B (100 U).

Antifungal susceptibility testing was performed by disk diffusion method using Mueller-Hinton Agar $+2 \%$ Glucose and $0.5 \mu \mathrm{g} / \mathrm{ml}$ Methylene Blue Dye (GMB) Medium as per CLSI guidelines (C.L.S.I. document M44-A2, 2009.). 0.5 McFarland standard was used to standardize the inoculums density. Candida albicans ATCC 90028 and Candida parapsilosis ATCC 22019 were used as quality control. The antifungal susceptibility of the isolates was interpreted as sensitive (S), dose dependent-susceptible (DDS) and resistant (R). The results were interpreted as per the Clinical and Laboratory Standards Institute (CLSI) guidelines [8].

\section{Results}

A total of 104 isolates of Candida species were obtained from different clinical specimens of all age group patients visiting the outpatient's department and in-patient department the period from June 2016 to June 2017. Of these 60 (58\%) were from Urine, 36 (34.5\%) were from Sputum and $8(7.5 \%)$ were from blood isolates.

Demographic data of patients: Of the 104 patients, 62 (54\%) were males and $42(46 \%)$ were females with the age group of $1-18=8(8 \%)$, $18-44=25(23.5 \%), 45-59=31(30 \%)$ and $>60=40(38.5 \%)$. The most common predisposing factor was diabetics [37(35.5\%)] followed by AIDS [4] (4\%)] respectively.

Of the total of 104 Candida species 64 (61.5\%) were Candida albicans, 20 (19\%) Candida dubliniensis, 10 (9\%) Candida tropicalis, 7(6.5\%) Candida parapsilosis and 4(\%) Candida glabrata respectively. We also found that a maximum number of blood isolates were from non-Candida albicans species [7(7\%)], and Candida albicans were most common among sputum and urine isolates. (Table 1)

\begin{tabular}{|l|l|l|l|}
\hline Candida species & Blood $\mathbf{n = 8}$ & Urine $\mathbf{n = 6 0}$ & Sputum $\mathbf{n = 3 6}$ \\
\hline Candida albicans $(\mathrm{n}=64)$ & $2(25 \%)$ & $33(55 \%)$ & $29(80.5 \%)$ \\
\hline Candida dubliniensis $(\mathrm{N}=20)$ & 0 & $15(25 \%)$ & $5(14 \%)$ \\
\hline Candida tropicalis $(\mathrm{n}=10)$ & $3(37.5 \%)$ & $5(8.5 \%)$ & $2(5.5 \%)$ \\
\hline Candida parapsilosis $(\mathrm{n}=7)$ & $2(25 \%)$ & $5(8.5 \%)$ & 0 \\
\hline Candida glabrata $(\mathrm{n}=3)$ & $1(12.5 \%)$ & $2(3 \%)$ & 0 \\
\hline
\end{tabular}

Table 1: Distribution of Candida species in different clinical isolates.

Resistance rates for commonly use antifungal drugs among the isolates are as followed Fluconazole 76 (73\%), Amphotericin B 40 (38.5\%), Nystatin 4(4\%) and Clotrimazole 60( 58\%) respectively. Prevalence of drug resistance among the Candida albicans, Candida dubliniensis and non-Candida albicans and non-Candida dubliniensis are summarized in Table 2 . 
Citation: Singh M, Chakraborty A (2017) Antifungal Drug Resistance among Candida albicans and Non-albicans Candida Species Isolates from a Tertiary Care Centre at Allahabad . J Antimicrob Agents 3: 150. doi:10.4172/2472-1212.1000150

Page 3 of 4

\begin{tabular}{|c|c|c|c|c|}
\hline Anti-fungal drugs & Candida albicans $(n=64)$ & Candida dubliniensis $(\mathrm{n}=20)$ & $\begin{array}{l}\text { Non- albicans \& } \text { non } \\
\text { dubliniensis Candida }(\mathrm{n}=20)\end{array}$ & Total $(\mathrm{N}=104)$ \\
\hline \multicolumn{5}{|l|}{ Fluconazole } \\
\hline Sensitive & $8(12.5)$ & $12(60)$ & $8(40)$ & $28(27)$ \\
\hline Resistance & 56(87.5)\# & $8(40)$ & $12(60)$ & 76(73)\# \\
\hline \multicolumn{5}{|l|}{ Amphotericin B } \\
\hline Sensitive & 48(75)\# & 16(80)\# & 0 & $64(61.5)$ \\
\hline Resistance & $16(25)$ & $4(20)$ & 20(100)\# & $40(38.5)$ \\
\hline \multicolumn{5}{|l|}{ Nystatin } \\
\hline Sensitive & 60(93.75)\# & 20(100)\# & 20(100)\# & 100(96)\# \\
\hline Resistance & $4(6.25)$ & 0 & 0 & $4(4)$ \\
\hline \multicolumn{5}{|l|}{ Clotrimazole } \\
\hline Sensitive & $12(18.75)$ & 16(80)\# & 16(80)\# & $44(42)$ \\
\hline Resistance & $52(81.25) \#$ & $4(20)$ & $4(20)$ & $60(58)$ \\
\hline
\end{tabular}

Table 2: Antifungal susceptibility pattern of Candida isolates.

\section{Discussion}

The epidemiology of Candida infection has changed in recent years. For many years Candida albicans were the most dominant species to cause invasive infection in most clinical settings, a considerable percentage of patients are now infected with non-albicans Candida species. Infections with these Candida species also have a direct impact on the choice of empiric antifungal therapy and clinical outcome as they show resistance to multiple antifungal drugs.

In the present study, we have found that among the study population maximum numbers of the isolates were from urine, which indicates that other than bacteria in our population Candida which also causes the substantial number of infection. Several other investigator form India also reported a high prevalence of Candida causing UTI [4]. However, the incidence of invasive isolates was less in our study isolates in compare to other studies where they had found a high prevalence of invasive isolates in their study population $[9,10]$.

Result of our study has shown that a maximum number of the isolates were from male patients and elder population were mostly effected which is with the agreement of the study done by Pahwa et al. [4] We are not the first to report that diabetics were one of the most common predisposing factors for candidiasis other investigator also reported the same [11]. These findings indicate that due to the poor immune response by elderly patients and predisposing factor such as diabetes mellitus may act as an important risk factor for candidiasis.

In the present study we observe that in our population with Candida albicans as well as non- albicans species were also causing infection, several other investigators also reported the same from the different part of the country $[4,9]$. A study done by Deorukhkar et al [12] showed a higher frequency of NAC (63.3\%) in comparison to Candida albicans (36.7\%). Yet another study by Bajwa and Kulshrestha have quoted that the isolation rate of NAC documented in India ranges from $52 \%$ to $96 \%$, with Candida tropicalis being the predominant species instead of Candida glabrata or Candida parapsilosis in all age groups. [13].

The antifungal susceptibility testing revealed that amphotericin B exhibited a higher sensitivity against Candida albicans, Candida dubliniensis respectively, which points out that this drug can serve as a good candidate for empirical therapy of Candida albicans and Candida dubliniensis infections. This finding is similar to the studies done by Kaur et al. [14] and Mondal et al. [15].

However, non-albicans and non-dubliniensis Candida were showing the high degree of resistance against Amphotericin B. The most active antifungal drugs for all the Candida species were Nystatin.

We also found that Clotrimazole was more active against nonalbicans species (80\%) which is similar to the study done by Ajitha et al. [16]. Where they have found around $67 \%$ of their non-albicans isolates were sensitive to clotrimazole. However, in the present study, we have found the very high label of Clotrimazole resistance among the Candida albicans isolates.

\section{Conclusion}

In conclusion, microbiology laboratories must be able to detect the different species of Candida and their resistance pattern in a timely manner, Microbiological excellence is needed more than ever, and the resistance should be detected accurately. In addition, there should be good communication between the microbiologist and the health care worker to make better patient outcomes, facilitating effective infection control, reducing spread of resistant pathogens and helping hospitals to meet accreditation standards.

\section{References}

1. Jarvis WR (1995) Epidemiology of nosocomial fungal infections, with emphasis on Candida species. Clin Infect Dis 20:1526-1530. 
Citation: Singh M, Chakraborty A (2017) Antifungal Drug Resistance among Candida albicans and Non-albicans Candida Species Isolates from a Tertiary Care Centre at Allahabad . J Antimicrob Agents 3: 150. doi:10.4172/2472-1212.1000150

Page 4 of 4

2. Chow JK, Golan Y, Ruthazer R, Karchmer AW, Carmeli Y, et al. (2008) Factors associated with candidemia caused by non-albicans Candida species versus Candida albicans in the intensive care unit. Clin Infect Dis 46: 1206-1213.

3. Pfaller MA, Diekema DJ, Gibbs DL, Newell VA, Ellis D, et al. (2010) Results from the ARTEMIS DISK Global Antifungal Surveillance Study 1997 to 2007: A 10.5-year analysis of susceptibilities of Candida species to fluconazole and voriconazole as determined by CLSI standardized disk diffusion. J Clin Microbiol 48: 1366-1377.

4. Pahwa N, Kumar R, Nirkhiwale S, Bandi A (2014) Species distribution and drug susceptibility of Candida in clinical isolates from a tertiary care centre at Indore. Indian J Med Microbiol 32: 44-48.

5. Campbell CK, Holmes AD, Davey KG, Szekely A, Warnock DW (1998) Comparison of a new chromogenic agar with the germ tube method for presumptive identification of Candida albicans. Eur J Clin Microbiol Infect Dis 17: 367-368.

6. Fisher F, Cook N (1998) Reagents, stains, media and methods. Fundamentals of diagnostic mycology. Saunders. Pp. 320-340.

7. Hospenthal DR, Beckius ML, Floyd KL, Horvath LL, Murray CK (2006) Presumptive identification of Candida species other than Candida albicans, Candida krusei and Candida tropicalis with the chromogenic medium CHROMagar Candida. Ann Clin Microbiol Antimicrob 5: 1-5.

8. Clinical and Laboratory Standards Institute (2009) Clinical and Laboratory Standards Institute Method for Antifungal Disk Diffusion Susceptibility Testing of Yeasts; Edition 2.
9. Sandhu R, Dahiya S, Sayal P, Budhani D (2017) Increased the role of nonalbicans Candida, potential risk factors, and attributable mortality in hospitalized patients. J Health Res Rev 4: 78-83.

10. Yapar $\mathrm{N}$ (2014) Epidemiology and risk factors for invasive candidiasis. Ther Clin Risk Manag 10: 95-105.

11. Casqueiro J, Casqueiro J, Alves C (2012) Infections in patients with diabetes mellitus: A review of pathogenesis. Indian J Endocrinol Metab 16: S27-S36

12. Deorukhkar SC, Saini S, Mathew S (2014) Non-albicans Candida infection: An emerging threat. Interdiscip Perspect Infect Dis 6: 159-158.

13. Bajwa S, Kulshrestha A (2013) Fungal infections in intensive care unit: Challenges in diagnosis and management. Ann Med Health Sci Res 3: 238-244

14. Kaur R, Dhakad MS, Goyal R, Kumar R (2016) Emergence of nonalbicans Candida species and antifungal resistance in intensive care unit patients. Asian Pac J Trop Biomed 6: 455-460.

15. Mondal S, Mondal A, Pal N, Banerjee P, Kumar S, et al. (2013) Species distribution and in vitro antifungal susceptibility patterns of Candida. J Inst Med 35: 45-49.

16. Reddy A, Mustafa M(2014) Phenotypic identification of Candida species and their susceptibility profile in patients with genitourinary candidiasis. Int J Adv Res 12: 76-84. 